\title{
Foreword
}

\section{Walter Boltz}

Trading on Europe's gas wholesale markets or at hubs has made progress over the last decade, particularly in Northwest Europe. However, progress has not been the same in all parts of Europe and even where gas hubs have emerged, liquidity is still regarded by many as insufficient. Nevertheless, more needs to be done for European gas wholesale markets to be effectively connected to each other and to be sufficiently liquid to send reliable price signals - or in other words - to meet our goal of the Internal Energy Market.

The idea of the integrated energy market is to promote competition in the European wholesale markets and facilitate new entry to compete against the incumbents in supplying gas. A competitive wholesale market will need to be efficient, thereby delivering gas to where it is most valued while providing shippers with the right incentives to secure supplies to European consumers. An efficient market must also provide the signals for investment in both gas production and in gas network infrastructure, including transmission, storage and LNG (liquefied natural gas) facilities, in order to meet the demands of European gas consumers. There is also a strong need for non-discriminatory and fair arrangements for shippers to access the gas infrastructure in order for competition to develop and for the network to be used efficiently while guaranteeing adequate remuneration for investments. We need a regulatory framework that secures supplies in the long, medium and short term, which means making Europe, now and in the future, attractive to gas imports and taking account of seasonal and short-term fluctuations in gas demand.

One of the key challenges in creating an integrated market in Europe is to put effective rules in place for facilitating cross-border trading and market integration between Member States. As such, the Third Energy Package establishes a new regulatory framework for cross-border trade and provisions for legally binding European network codes to regulate cross-border aspects.

The European regulatory framework aims to improve efficiency in the use of cross-border capacity and to enhance competition through 
liquid wholesale markets. As part of the Third Energy Package the Gas Regulation ${ }^{1}$ makes Transmission System Operator (TSO)-wide entry/exit systems obligatory by September 2011 and abolishes tariffs set on the basis of contract paths. ${ }^{2}$ Measures are being pursued to open up network access to new entrants in a bid to foster greater competition. The Gas Directive ${ }^{3}$ requires increased unbundling of the ownership and operation of gas networks from gas supply in order to remove potential for discrimination when granting network access.

European energy regulators have prepared, together with stakeholders, a vision for the European Gas Market with a view to completing the Internal Energy Market by 2014 as requested by the European Council and the European Commission. In our vision we suggest there be a series of liquid hubs across Europe where gas can be traded (these may be national or across borders) and that national markets are easily connected to these trading points. Gas will flow freely between these market areas and respond to price signals, that is, gas will flow to where it is valued most. It will also be important that sufficient and efficient levels of infrastructure investment take place to allow markets to integrate.

\section{NOTES}

1. Regulation (EC) $715 / 2009$.

2. Recital 19 Gas Regulation.

3. Directive 2009/73/EC. 